\title{
RECENT ADVANCES IN THE PATHOLOGY OF CHRONIC ARTHRITIS AND RHEUMATIC DISORDERS
}

\author{
By Douglas H. Collins, O.B.E., M.D., M.R.C.P. \\ Joseph Hunter Professor of Pathology in the University of Sheffield
}

The word rheumatism, except in its limited sense of acute rheumatism or rheumatic fever, has no meaning in pathology. The rheumatic diseases are a heterogeneous group of morbid states, mainly affecting the locomotor system and most of them painful, chronic and of low mortality. To this major group, certain minor groups of ailments are from time to time added, or subtracted, according to the bias of current medical thought and the expediences of current medical practice. The last large subtractions have been most of the painful peripheral neurological disorders, and the latest additions are certain dermatological diseases with systemic effects which are supposed to resemble rheumatoid arthritis in their basic pathology.

The fact remains, however, that most of the rheumatic disorders have nothing in common pathologically except that they affect the more or less specialized mesenchymal tissues of the joints and other parts of the locomotor apparatus, and that the cause of each, if not completely unknown, is imperfectly understood. The chronicity of the diseases, their dependence upon largely subjective manifestations for their detection in the early stages, the limited reaction patterns of connective tissues and the impossibility of reproducing in animals the identifiable counterparts of the human ailments, all hinder the pathologist in searching for the causes and origins of rheumatic disease. Nevertheless, his studies have materially helped in separating the different forms of disease and in explaining some of their clinical characteristics.

Much the most important of the chronic rheumatic disorders are the two contrasting forms of chronic arthritis, osteoarthritis and rheumatoip arthritis. Each disease has pathological features of great interest, which have formed the field of many recent researches, and they alone will be discussed in detail in what follows.

\section{Osteoarthritis}

A lesion of articular cartilage is now generally held to be the initial lesion of osteoarthritis. In most cases, this is a gradual and progressive loss of depth resulting from attrition of cartilage which through ageing or other causes has become less resilient and less resistant to mechanicalo influences. Occasionally, direct injury, fracture $\vec{\omega}$ involving the joint surfaces, hard loose bodies, or previous inflammatory joint disease may damageo previously healthy articular cartilages and initiates a rapidly developing osteoarthritis, just as Ax-은 hausen (I925), 30 years ago, observed the development of marginal osteophytes and 'arthritis deformans' in the joints of the rabbit and the dog after cauterizing or otherwise injuring the cartilaginous surfaces.

Articular cartilage comprises a tracery $\Phi f^{5}$ collagen fibres springing from the underlyiges bone and set in a matrix of which the chief cosi ponent is the mucopolysaccharide, chondroitmp sulphate A (Meyer, 1954). The cartilage cellss apparently play little or no active part in itso pathological changes which are essentially de-ڤ generative. Cartilage has only a feeble capacity for repair, and it seems that the existence of suitable matrix is necessary before any multi-3 plication of cartilage cells can take place (Subbar Rao, 1954). It is this virtual irreversibility of degeneration and destruction of cartilage, onces begun, that allows the development of the fulB. cycle of osteoarthritic changes in a joint. Traumad can initiate the process, but in general the disease is so closely linked with age that we must supposeo that involutional changes in cartilage structure permit its wearing down by the ordinary use of the joint. Although fissuring of cartilage has been observed in certain areas of the hip joint not exposed to pressure or friction (Harrison et al. 1953), the cartilage lesions really associated with ${ }^{\circ}$ osteoarthritis are always first seen on areas of the joint surface exposed to friction, weight-bearing or great movement. The collagen fibres in cartilage form an arcade towards the surface, and the earliest changes take the form of a tangentia $\bar{D}$ flaking off of the surface zone (Fig. I). Later? the cartilage cracks along the perpendicularo collagen-fibre lines, a process known as fibrilla 


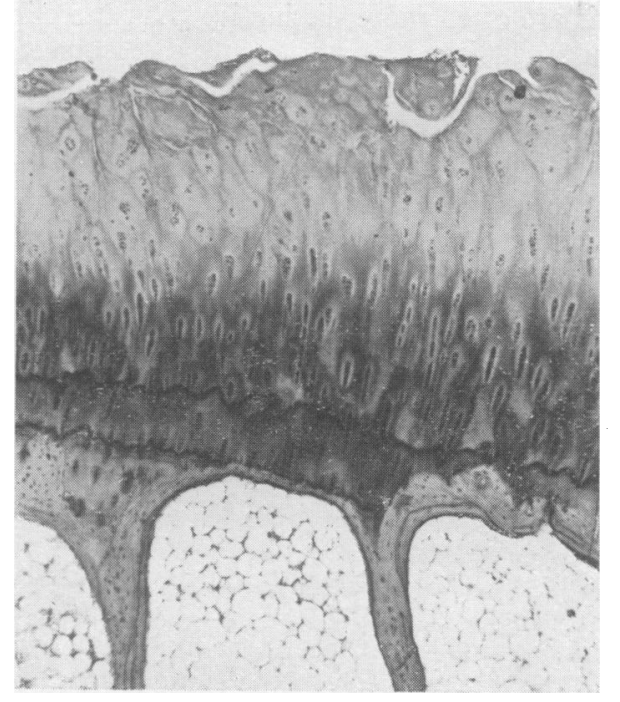

Fig. r.-Osteoarthritis. Early cartilage lesion. Tangential flaking of surface layers. Doubling of the deep calcified line, as here, is not a constant feature. Haematoxylin and eosin $\times 20$.

tion, which in time extends to the underlying bone and is accompanied by the death of the cartilage cells (Fig. 2). Both histological appearances and chemical analyses (Matthews, 1953) indicate a degradation and loss of chondroitin sulphate matrix in advance of actual mechanical attrition. It is this that justifies the American use of the term 'degenerative arthritis' for osteoarthritis. But almost everything else that follows is regenerative or formative on the part of the bony and fibrous tissues of the joint.

Cartilage degeneration and destruction must both operate together in leading to an alteration of contour of the articular surfaces. This is met by an adaptation of form on the part of the bone and synovial and capsular tissues of the joint, which, unlike cartilage, retain the power of regeneration, repair and remodelling. The evolution of osteoarthritis follows its own course in any particular joint, the structural adaptations there being dependent upon the changing mechanical conditions. As cartilage degenerates, the normal stability of the chondro-osseous junction is lost, and ossification advances into the proper territory of cartilage. Some marginal osteophytes are formed this way; others result from intramembranous ossification at the points of periosteal attachment of the vascular synovial membrane. Sclerosis of the subchondral osseous plate and a re-alignment of the trabeculae of the cancellum may also occur. The capsule usually becomes fibrotic, and inflammatory changes in the synovial

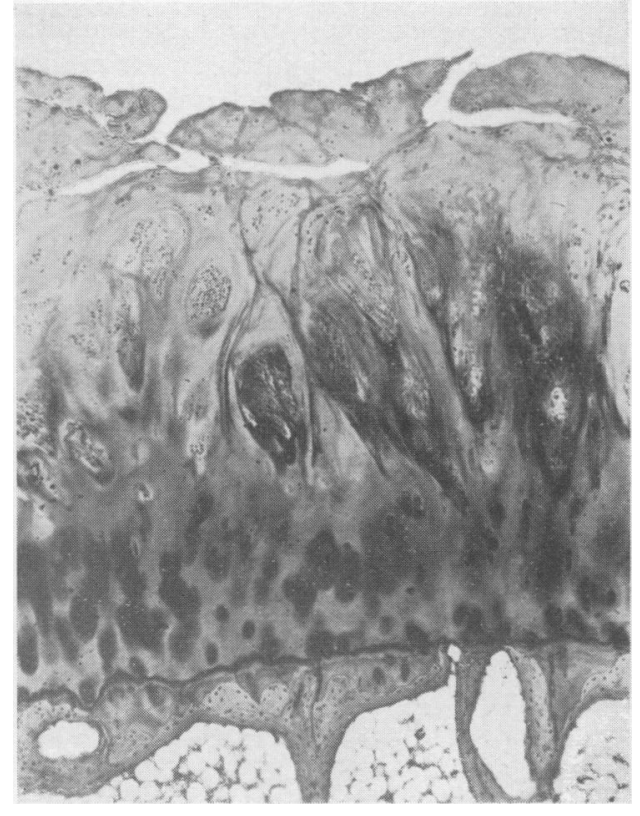

FIG. 2.-Osteoarthritis. Later cartilage lesion. Fibrillation extends down the vertical fibres and various degenerative changes in the cartilage cells are also seen. H. and E. $\times 20$.

membrane are minimal and generally confined to a mild reaction around engulfed particles of dead bone or cartilage. The extent of the synovitis is of the same order as that seen after simple traumatic injury to the joint, and when there is an effusion it is viscous and clear with a total nucleated-cell count usually well below 5,000 per c.mm. and polymorphonuclear leucocytes rarely more numerous than 20 per cent.

Over the age of 50 , cartilage changes are seen on naked-eye examination in about 90 per cent. of knee joints and of acromio-clavicular joints, in the majority of hallux, elbow and hip joints, and in a lesser proportion of sterno-clavicular and shoulder joints (Heine, 1926). Advanced changes in cartilage with some remodelling of the bone ends and osteophytic outgrowths are to be found at necropsy on persons over 50 in about 25 per cent. of knee joints, rather less frequently in the hallux and acromio-clavicular joints, in about Io per cent. of hip and elbow joints, less commonly in the shoulder and very rarely in the sterno-clavicular joint. These figures are strong arguments for the view that osteoarthritis is the projection to a pathological degree of the age changes which develop in functioning joints and that local mechanical conditions in each joint determine its development. Local mechanical conditions also certainly determine its degree and 
probably its age of onset, for local conditions which expose a particular joint to undue mechanical stress lead to an early and quickly evolving osteoarthritis. Examples of such conditions are old rickety deformity, genu valgum, coxa vara, Paget's disease, paralysis of the opposite limb, old fracture and old rheumatoid or infective arthritis.

Viewed from the standpoint of pathogenesis, it seems evident that the principle therapeutic aim, apart from symptomatic relief, must be so to stabilize the mechanical conditions operating at the affected joint that no further deformation of the joint results.

Up to now I have stressed the local nature of osteoarthritis. In discussing the possible systemic backgrounds of osteoarthritis we are on more debatable ground. Senile involution of connective tissues must play a part. Obesity is an aggravating factor. Osteoarthritis may clinically affect many joints simultaneously, a fact which is not surprising, since at necropsy of any old person one may find the disease in various subclinical forms in most joints.

The radiographic features of osteoarthritis can be closely correlated with what has been found by anatomical dissection and histology. What is usually called ' loss of joint space' is, in fact, loss of cartilage depth. Marginal osteophytes result from advancing ossification at disturbed chondroosseous junctions or from periosteal new bone formation. Moulding of the bone ends is due to remodelling of the plastic living bone, by resorption and regeneration, under the stimulus of changing mechanical forces, and may be interpreted as an attempt to maintain congruity of the articular surface. Cysts often seen in the bone ends in osteoarthritis have been shown histologically to communicate with the joint cavity and may arise from the propulsion of synovial fluid into the cancellous bone (Landells, 1953).

By definition, osteoarthritis is a disease of synovial joints. The mere demonstration of osteophytic spurs which may arise by ossification into tendinous or fascial attachments, or in areas of periosteal elevation, does not constitute the diagnosis of osteoarthritis. There is still some doubt whether all forms of Heberden's nodes (Stecher, I955) really represent osteoarthritis. The wellknown condition of osteophytosis of the vertebral bodies, or spondylosis deformans, is not a true osteoarthritis, since diarthrodial joints are not affected. It arises from changes in the intervertebral discs in a manner which has been fully described elsewhere (Collins, 1949, p. 3॰5).

\section{Rheumatoid Arthritis}

The pathological features of rheumatoid ar- thritis are often more distinctive than the clinical. $\frac{\pi}{\infty}$ As opposed to osteoarthritis the disease seems to be one of systemic distribution in which the $e^{c}$ affection of the joints constitutes the major but not the whole part, and the tissue changes areo those of subacute and chronic inflammation. These are accompanied in some situations, butên not everywhere, by focal degeneration of fibrous tissue, a relatively minor feature in the morpho- 0 logical pathology of the disease which has led to its inclusion within that nosological chimera, thẹ so-called collagen diseases.

Foints. Rheumatoid arthritis starts in the joints as an inflammatory synovitis characterizedo by hyperaemia, exudation and leucocytic reaction shown, respectively, by the increased heat of the joint, by oedema of the synovial tissues and joint $\vec{\omega}_{\omega}$ effusion, and by the presence of polymorpho- N nuclear leucocytes in the joint fluid and lymphom cytes and plasma cells in the synovial membrane An inflammatory effusion is always present in the early and active stages of the disease in both small and large joints. It contains sufficient cells, on the average 20,000 per c.mm., to make if slightly turbid, and most of these cells, often ups to 90 per cent., are neutrophil polymorphs. the inflammation does not proceed to suppuration, unless, as occasionally happens, the joint beconfes secondarily invaded by pyogenic organisms. does the inflammation readily resolve. It p $\overline{\mathrm{er}}$ sists in a subacute phase, and proliferation of the synovial connective tissue produces hyperplastig synovial granulation tissue and a form of diffuse granuloma within the joint. The synovial mem $\frac{1}{2}$ brane becomes thickened by new fibroblasts $\overrightarrow{\vec{F}}$ fibrocytes and histiocytes, more richly vascular and thrown into folds. Enlarged or newly formed villi, covered by a greatly increased number of more or less well differentiated synovial lining cells, crowd into the joint cavity. At the same time, the hyperplastic synovial tissues start to creep across and attach themselves to the articular. surfaces, clothing them in a pannus (Fig. 3 ) The infiltrating leucocytes, as is the rule in non? suppurative but persistent inflammations, are mainly lymphocytes, but sometimes more promi nently plasma cells, congregating first around the vessels whence they have come, and when numerous forming follicular aggregates in the subsynovial layer and villi (Fig. 4).

Kulka et al. (1955) have recently studied th\& histological character of the synovial lesions in the early course of rheumatoid arthritis. The domio nant feature was always a diffuse proliferative synovitis. Lymphocytic infiltration was oftef light and follicles seldom prominent. A fibrinoid type of exudate was particularly noticed in these्త early lesions, but histochemical tests were no 


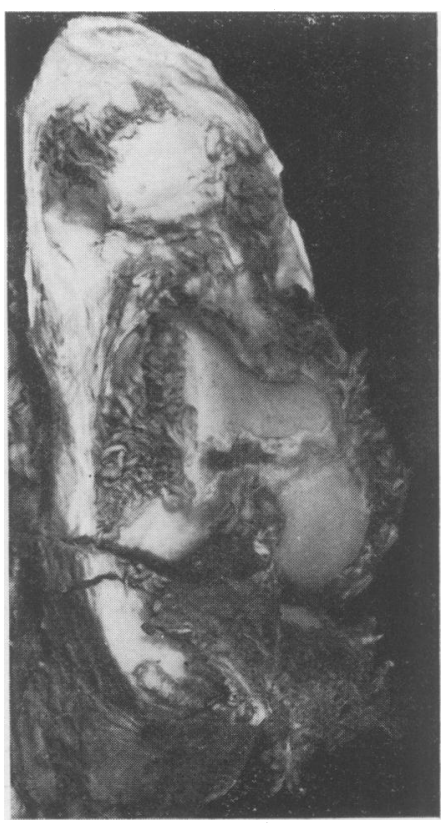

FIG. 3.-Rheumatoid arthritis. Chronically affected knee-joint, opened with patella reflected upwards. Note the hyperplastic granulomatous synovial tissues with great numbers of villi. The pannus lying over the tibial condyles also adhered to the femoral cartilage which shows erosion just above the intercondylar notch.

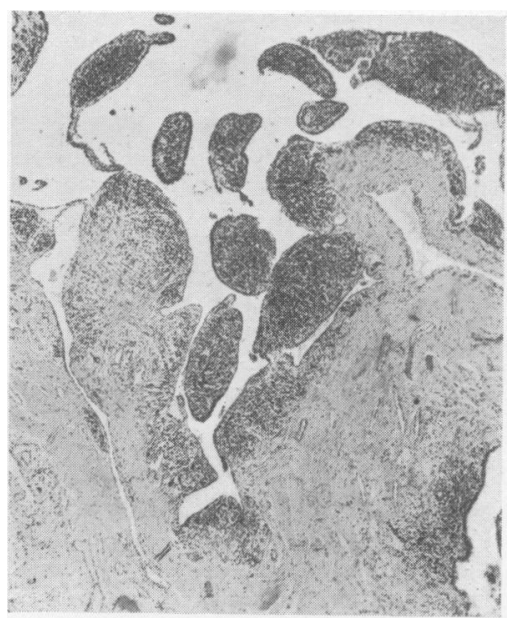

Fig. 4.-Rheumatoid arthritis. Characteristic synovitis. Proliferation of all synovial connective tissues with dense lymphocytic or plasma-cell infiltration often forming compact aggregates in the bulbous and enlarged villi. H. and E. $\times 20$.

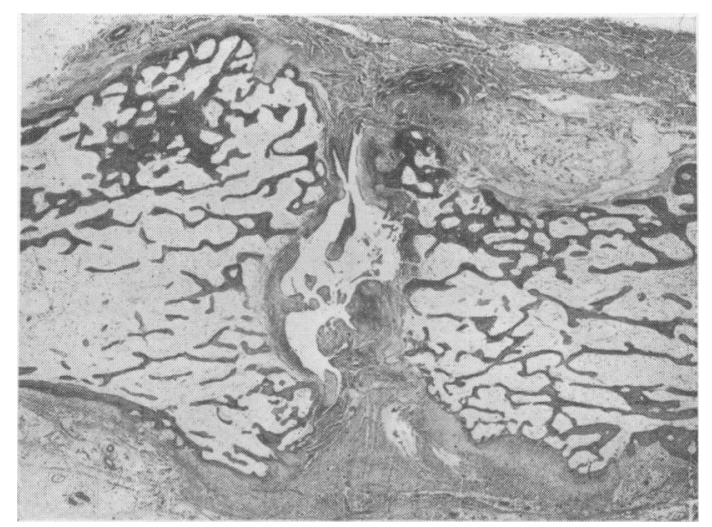

Fig. 5.-Rheumatoid arthritis. Advanced disease of a $\stackrel{\omega}{\vec{\omega}}$ proximal interphalangeal joint. Granulation tissue $\omega^{\prime}$ has grown into the joint, spreading as a pannus over $\mathrm{N}$ both cartilages, and eroding much of the head of the proximal phalanx (right). Only a small island of $\mathrm{N}$ articular cartilage remains, at the top of the cavity 0 and on the right (proximal) side. The bone is atrophied. The joint was fixed by capsular and intracapsular fibrosis. $H$. and E. $\times 3.5$.

specific, and rather similar appearances are see in other forms of arthritis and in fibrinous inflane mations generally. In my own experience, an expansion of a synovial or bursal cavity take. place by a process resembling fibrinoid degenerat tion, and this appearance has little of rheumatic specificity about it except when it forms part of the whole characteristic picture of the rheumatic or rheumatoid granuloma.

The granulomatous reaction, starting in the $\overrightarrow{\vec{A}}$ synovial membrane, progressively involves the $\frac{0}{3}$ joint more and more extensively. The articular cartilages become eroded by adherent pannus, an effect which may result from histolytic activity, $\overline{0}$ perhaps enzymic, on the part of the cells in the 3 . granulation tissue or from intrinsic degeneration $\frac{\sigma}{3}$ of matrix. The bone-ends also become invaded by granulation tissue arising in the periosteal reflection of the synovial membrane, and this accounts for the small nibbled-out rarefactions so 웅 often seen radiographically in the bones forming $\rightarrow$ a severely affected rheumatoid joint. It is this relentless progression of erosion of previously $\tilde{N}$ normal structures by granulation tissue that leads to the ultimate gross deformities of the joints with

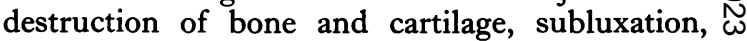
intra-articular adhesions and capsular fibrosis (Fig. 5).

A severe degree of bone atrophy is usual in the region of an affected joint. Much, but perhaps not $\stackrel{\odot}{+}$ all, of this can be explained on a basis of disuse $T$ atrophy, for local atrophy of bone can proceed very rapidly from disuse and becomss especially 


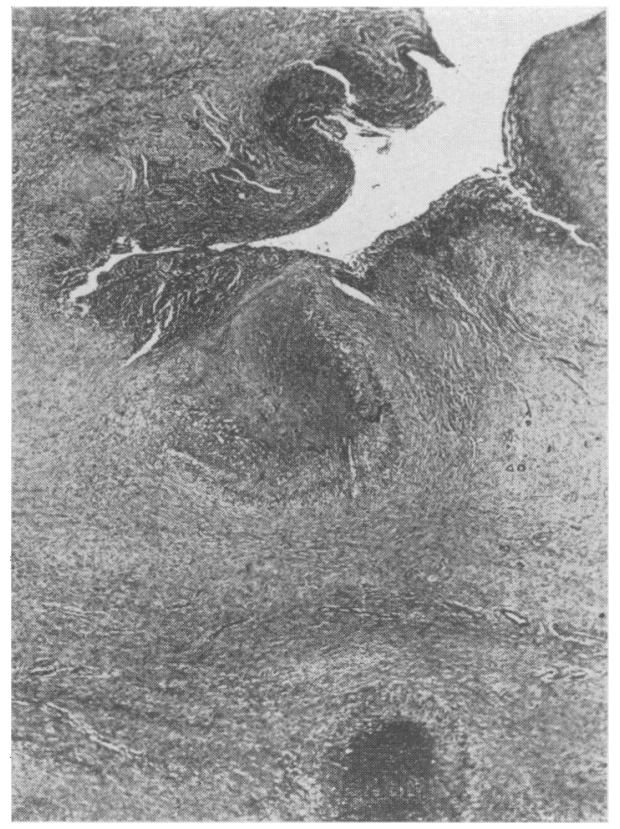

FIG. 6.-Rheumatoid arthritis. Tenosynovitis. The stratum synoviale near the cavity of the tendon sheath is infiltrated by lymphocytes. All tissues are proliferated and the sheath is itself greatly thickened. Two necrotic foci with marginal palisading of cells, resembling the structure in Fig. 8 , are seen, one just below the synovial surface and the other much more deeply placed. Trichrome $\times 20$.

obvious where the bones are mainly cancellous, as in most epiphysial areas. In addition, there are excavations of bone by invading inflammatory tissue, and perhaps by independent deeply situated foci of inflammatory osteitis.

Tendons and tendon-sheaths. Tenosynovitis is not uncommon in rheumatoid arthritis and takes the same form as articular synovitis, except that for some reason centres of fibrinoid necrosis are more often encountered in the granulomatous synovium of tendon-sheaths than of the joints (Fig. 6). Buried in the deeper parts of the synovial tissues they histologically resemble small subcutaneous nodules. Near the cavity of the tendon-sheath they may discharge their fibrinoid necrotic centres, which if inspissated and not broken up may form melon-seed bodies just like those produced by the analogous process of caseation in tuberculous tenosynovitis. Rheumatoid nodules sometimes develop within the collagenous substance of the tendons themselves and may cause great thickening and nodularity (Kellgren and Ball, 1950) and sometimes their spontaneous rupture (Harris, I95I).

Disseminated lesions. Until fairly recently the subcutaneous nodules were the only extra-

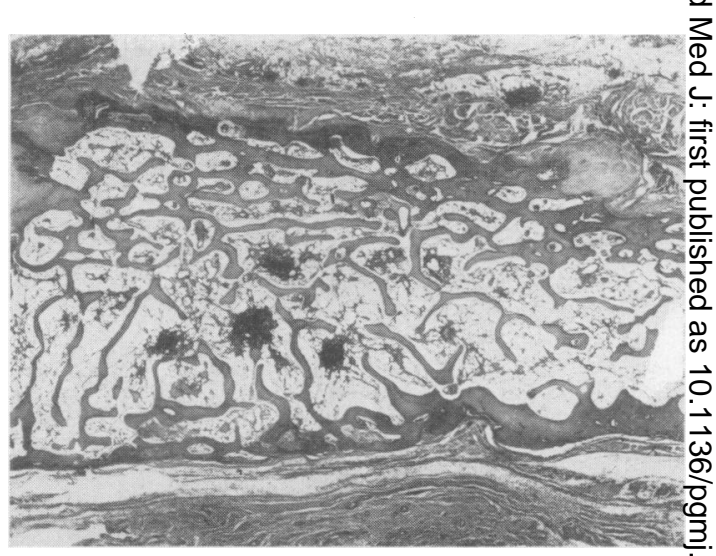

$\omega$

FIG. 7.-Rheumatoid arthritis. Disseminated lymphoid foci in bone marrow (acromial process) and ino investing fascia and muscle. H. and E. $\times 20$.

articular lesions recognized as part of the rheu-음 matoid arthritis picture, but pathological lesions, as a rule clinically silent, are present in many, situations, especially in the more severe and chronic case. These are of two kinds: (a) micre-3 scopical inflammatory foci or lymphorrhages, and (b) nodules, often visible to the naked eye, whigh $\vec{c}$ include one or more centres of collagen degenets-s tion. In addition to these two kinds of discrese lesions there are often certain generalized systemico disturbances-lymphoid hyperplasia of a reactive lymphadenopathy type (Motulsky et al., 1952), splenomegaly, neutropenia, anaemia, hyperglo-命 bulinaemia and amyloidosis.

Disseminated inflammatory or lymphoid foci. $\overrightarrow{0}$ These consist mostly of lymphocytes, but may3 contain also a few histiocytes, fibroblasts or? plasma cells. They have been found in the interstices of voluntary muscles, not necessarilyo wasted or related to affected joints, and in the sheaths of peripheral nerves (Cruickshank, 1952). $\frac{\text { - }}{3}$ They may be found also in the bone marrow, periosteum and fascia generally (Fig. 7). Some-O times the lymphorrhages are related to blood vessels. Distinctly inflammatory focal arteritis of the necrotizing angiitis type (Zeek, 1952) has also been found in rheumatoid arthritis (Cruickshank, 1954). These small lesions can seldom be related to clinical features; they are the fruit of pathological searching. The very fact that lesions of this type are not specific to the disease carries $\omega$ some significance, for they may be found also in rheumatic fever, dermatomyositis, lupus erythematosus and in hypersensitivity animal experiments. They may be the index, not of the ${ }^{\text {? }}$ widespread action of virus or toxin, but of an뭄 acquired hypersensitivity of the tissues in rheu- $\frac{\text { के }}{D}$ matoid arthritis. 


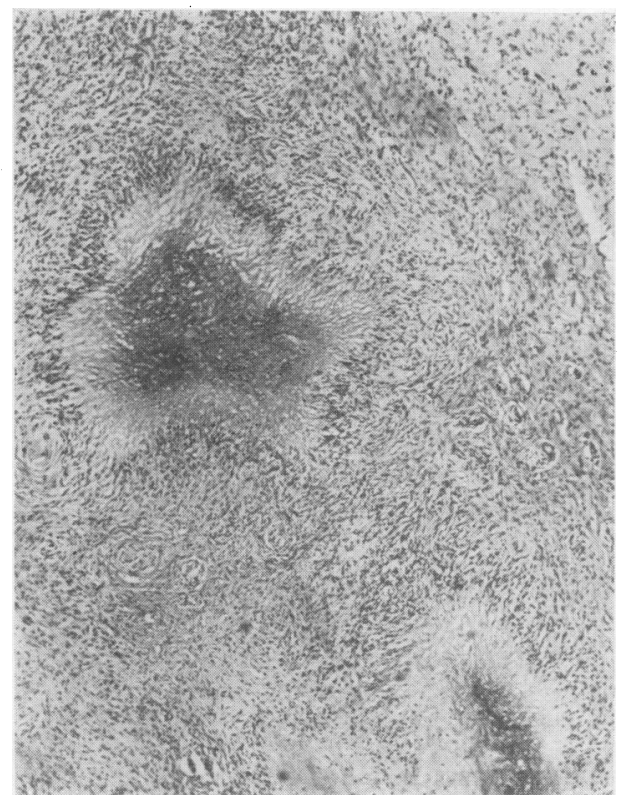

FIG. 8.- Rheumatoid arthritis. Subcutaneous nodule. Characteristic necrotic centres with palisade of fibroblasts and histiocytes. H. and E. $\times 30$.

Nodules. The subcutaneous nodules of rheumatoid arthritis have been studied for many years (Collins, 1937) and their structure is now well known. The central area of necrosis, I believe, develops within a new formation of connective tissue which has arisen as a granuloma at a site of mild repeated trauma. The expanding necrotic centre is surrounded by a palisade of fibroblasts and histiocytes (Fig. 8), and outside this is a cellular fibrous tissue rich in small blood vessels and diffusely infiltrated by lymphocytes and plasma cells. Bennett (1953) has remarked that the overall histological picture is not unlike a syphilitic gumma, although not implying thereby any common pathogenesis. There is some truth in the simile, for both are formative granulomatous lesions in which centres of necrosis appear, but the relative parts played by histolytic agents and by ischaemia in the production of necrosis in the rheumatoid nodule are unknown.

A most interesting recent development has been the discovery of morphologically typical rheumatoid nodules in other connective tissues, especially those where compact hyalinized collagen already exists. Nodules in the substance of tendons have already been mentioned. Nodules have been seen occasionally in scarred heart valves and annulus fibrosus (Baggenstoss and Rosenberg, 1944), in cranial dura mater, sclera of the eye, hyalinized splenic capsule and peri- cardium (Ellman et al., I954; Maher, 1954), and recently Gough et al. (I955) have illustrated a rheumatoid nodular reaction around collagenous foci in the lungs of coal miners with rheumatoid arthritis.

Rheumatoid arthritis and the 'collagen diseases.' There is today a widespread belief in the existence of a pathological monster called ' collagen disease,' like the chimera an apparently integrated being, although composed of incongruous parts. This popular term was introduced by Klemperer and his colleagues largely in consequence of their study of the lesions in systemic lupus erythematosus. It is too seldom remembered that in linking together clinically distinct diseases such as rheumatic fever, rheumatoid arthritis, disseminated lupus erythematosus and generalized scleroderma there was no intention of attributing a common aetiology to them (Klemperer, 1947). The idea was proposed in order ' to call attention to the significance of the connective tissue as the site of morbid changes and to invite investigations of the reasons for its alteration' (Klemperer, I 954). If it is indeed helpful to seek pathological similarities rather than dissimilarities amongst these diseases, then it is better to speak of 'collagen diseases,' to avoid the implication that there is an all-embracing pathogenic state of 'collagen disease.' A realistic attitude to what is implied may be engendered by speculating on whether? the necrobiotic lesions of tuberculosis and syphilis or the dermal and vascular lesions of diabetes would have been designated collagen disease had the responsible aetiological agents not already been known.

The concept of collagen diseases originated not only as an extension of, but as a corrective to, Klinge's (1933) assertion that fibrinoid swelling of connective tissue was the common basis of the lesions in rheumatic fever, rheumatoid arthritis and certain other diseases, indicating that they all had an allergic background and were based upon hypersensitivity reactions in the tissues. Klemperer, it appears, introduced a new term in order to avoid the implication that those diseases with disseminated focal lesions of collagenous connective tissues were necessarily dependent upon hypersensitivity or allergy. Some of this group certainly are so dependent. Rheumatic fever is a manifestation of a special form of acquired sensitivity to group A Str. pyogenes, and there is very strong evidence that polyarteritis nodosa and some other forms of necrotizing angiitis are hypersensitivity reactions. But the evidence is less convincing in the case of disseminated lupus erythematosus and very difficult to assess in the case of rheumatoid arthritis.

It is best to admit that no explanation yet 
advanced of the pathogenesis of rheumatoid arthritis tallies with all the evidence we have about its course and its pathological features. In short, the major lesions, those within the joints, are inflammatory and granulomatous. In the early stages fibrinous exudation is prominently associated with diffuse proliferative synovitis. That the initial lesions of the disease are of the collagen-degeneration type is a hypothesis open to question, and the view that disintegration products of collagen may account for the various toxic and inflammatory features of rheumatoid disease (Kellgren, 1952) still awaits proof. The disseminated nodular lesions showing well-defined focal necrosis of connective tissue are usually later manifestations and might be a measure of hypersensitivity acquired during the progress of the disease. It is disappointing that the introduction of adrenocortical hormones has done so little to elucidate the pathogenesis of rheumatoid arthritis, although it has led to many important discoveries about tissue reactions in general.

\section{Summary}

What are now called the rheumatic disorders are a group of pathologically diverse diseases, unified only by their mode of clinical presentation, and by the facts that they affect the locomotor system and that they are painful, chronic and mostly of unknown aetiology.

Recent advances in our pathological knowledge of the two major forms of chronic arthritis, osteoarthritis and rheumatoid arthritis, serve still further to distinguish between them.

In osteoarthritis, we do not yet know the precise conditions under which the initial changes occur in articular cartilage, but the ensuing changes can be explained by a mechanistic concept, having regard to the powers of repair and reconstruction with which the various connective-tissue structures are endowed.

In rheumatoid arthritis, the major lesions in the joints are inflammatory and granulomatous Disseminated lesions in many other parts of the body are of two main kinds: (a) microscopic inflammatory foci or lymphorrhages, $(b)$ nodulap lesions, often visible to the naked eye, which include one or more centres of connective-tissue degeneration or necrosis. In addition, there are often generalized systemic disturbances, such lymphoid hyperplasia, hyperglobulinaemia and amyloidosis. The position of rheumatoid arthritis in relation to the hypothesis of " collagen diseases. has been briefly discussed in view of the mis interpretations which the free use of such phrase is likely to engender.

\section{Acknowledgments}

I am very grateful to many colleagues various parts of the country for sending m material, and to my chief technician, Mr. A. W Collins, for the photographs.

\section{BIBLIOGRAPHY}

AXHAUSEN, G. (1925), Virchows Arch., 255, 144. BAGGENSTOSS, A. H., and ROSENBERG, E. F. (1944), Arcto

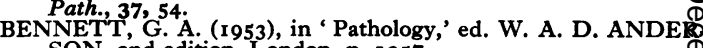
SON, 2nd edition, London, p. 1257.

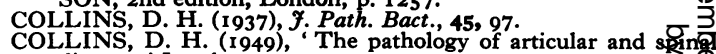
diseases,' London.

CRUICKSHANK, B. (1952), Y. Path. Bact., 64, 21.

CRUICKSHANK, B. (1954), Ann. Rheum. Dis., 13, 136.

ELLMAN, $P$ CUDKOWICZ, L

F. Clin. Path., 7, 239.
GOUGH, J., RIVERS, D., and SEAL, R. M. E. (1955), Tho IO, 9.

HARRIS, R. (1951), Ann. Rheum. Dis., 10, 298.

HARRISON, M. H. M., SCHAJOWICZ, F., and TRUETA (1953), f. Bone ft Surg., 35B, 598.

HEINE, J. (1926), Virchows Arch., 260, 521 .

KELLGREN, J. H. (1952), Brit. med. F., i, 1152. KLEMPERER, P. (1947), Bull. N.Y. Acad. Med., 23, 591.

KLEMPERER, P. (1954), in 'Connective tissue in health and disease,' ed. G. ASBOE-HANSEN, Copenhagen, p. $25 \mathrm{I}$.

KLINGE, F. (1933), Ergebn. allg. Path. u. path. Anat. 27,

KULKA, J. P., BÖCKING, D., ROPES, MARIÄN W. 1 ., BAÚER, W. (1955), Arch. Path., 59, 129.

LANDELLS, J. W. (1953), 买. Bone ft Surg., 35B, 643.

MAHER, J. A. (1954), Arch. Path., 58, 354.

MATTHEWS, B. F. (1953), Brit. med. $\mathcal{F}$., ii, 660.

MEYER, K. (1954), in 'Connective tissue in health and diseasę, MOTULSKY, A. G., WEINBERG, S., SAPHIR, O., and ROSEI BERG, E. (1952), Arch. int. Med., 90, 660.

STECHER, R. M. (1955), Ann. Rheum. Dis., I4, I.

SUBBA RAO, K. V. (1954), Ұ. Path. Bact., 67, 455.

ZEEK, PEARL M. (1952), Amer. $\boldsymbol{f}$. Clin. Path., 22, 777.

\section{HOW
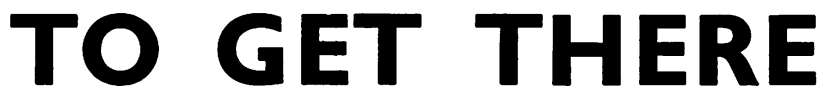

An Address Book for the Medical profession, showing how to reach the various Colleges, Societies, Institutes and Hospitals in or near London

New (Fourth) Edition: 1954

Price 2s. 6d. (2s. 9d., post free)

Published by the

FELLOWSHIP OF POSTGRADUATE MEDICINE

60 Portland Place, London, W.I 